NOTA TECNICA

\title{
ESTUDIO DE LA ESTABILIDAD EN LÍNEAS AVANZADAS Y VARIEDADES DE ARROZ (Oryza sativa L.) USANDO DOS METODOLOGÍAS1
}

\author{
Guillermo Castañon ${ }^{2}$
}

\begin{abstract}
RESUMEN
Estudio de la estabilidad en líneas avanzadas y variedades de arroz (Oryza sativa L.) usando dos metodologías. En Primavera-Verano de 1982 y 1983 se establecieron ensayos de arroz en euatro localidades del estado de Campeche, Méx. para estudiar la estabilidad de las varia-bles días espigamiento (DE), altura de planta (AP) y rendimiento de grano (RG). Dos metodologías fueron comparadas (Eberhart y Russell, 1966 y el de Wricke, 1965). La comparación fue con el objetivo de determinar cual método es más efectivo para detectar la estabilidad de los genotipos ensayados. En los resultados se encontró que todos los genotipos evaluados fueron diferentes o se vieron afectados por las condiciones ambientales de cada localidad de prueba. La media de AP es un poco reducida para la que deben poseer los eultivares de secano. Pero esta reducción se debió en gran parte al efecto del am-biente Edzná, que fue el más seco por el tipo de suelo no hidromórfico. Entre los genotipos ensayados existe potencial para RG en dos de las líneas avanzadas, estas son IR2055-466-6-6 e IR2055-48 1-2-1 Cu. De ellas alguna puede liberarse como nueva variedad para temporal, con la certeza de que dicho cultivar tendrá buen comportamiento. Fue indistinto el uso de cualesquier método para estudios de parámetros de estabilidad. Es decir se obtuvieron los mismos resultados con una o con otra metodología. Aunque cabe hacer la aclaración que el método propuesto por Wricke (1965) es por mucho más sencillo y de más fácil interpretación que el de Eberhart y Russell (1966).
\end{abstract}

\begin{abstract}
Stability study on rice (Oryza sativa L.) advanced lines and varieties. Four loealities in the State of Campeche, Mexico were used during the Spring-Summer of 1982 and 1983 to conduct trial in orferto study the stability of rice genotypes. The studied variables were: days to heading $(\mathrm{DH})$, plant height $(\mathrm{PH})$ and grain yield (GY). Two methodologies (Eberhart and Russell, 1966 and Wricke, 1965) were compared to determine which was most effective in detecting the stability ofthe tested genotypes. The results show that these tested genotypes were different or were environmentaly affected at each locality. The PH mea $n$ was slightly shorter for dry farming cultivars. This shortage was largely due to thc effect ofthe Edzna environment which was drier because of the soil type. Among the assayed genotypes, there is a potential for GY in two of the advanced lines (IR2055-466-6-6 and IR2055-481-2-1 Cu). One of these could be released as a new variety for rainfed conditions. The results show that either method can be used, giving the same response, although the Wricke's method is more easily interpreted that Eberhart and Russell.
\end{abstract}

\section{INTRODUCCIÓN}

Es muy importante estudiar la interacción genotipoambiente en la investigación agrícola para no cometer errores en la elección de la variedad superior que se quiera recomendar para una área agrícola amplia o específica.
Lo anterior trae como resultado la necesidad de que se establezcan experimentos repetidos tanto en tiempo como en espacio.

Yates y Cochran (1938) generaron la metodología para el análisis de experimentos mediante regresión con sitios, años y variedades.

\footnotetext{
1 Presentado en la XXXIX Reunión Anual del PCCMCA en Guatemala, C. A. 1993.

2 Proyecta del Programa de Mejoramiento Genético de Arroz. C. E. Campeche. Ave. José López Portillo W8. Campeche, Campo México. Actualmente Investigador del Sistema producto Maíz, C. E. Coxtaxtla. Apdo. Postal N 429. Veracruz, Ver. México.
} 
Los autores citados incluyeron análisis de regresión entre el rendimiento promedio de cada variedad por localidad con los promedios de rendimiento de las localidades evaluadas.

Años más tarde, Finlay y Wilkinson (1963), aplicaron el método de regresión lineal del rendimiento con el rendimiento de las variedades probadas para cada localidad y temporada, para así evaluar la adaptación de los cultivares ensayados.

La metodología más usada en el estudio de la interacción genotipo-ambiente es la propuesta por Eberhart y Russell (1966). Empero (Cruz y Salazar, 1992) señalan que este procedimiento generó controversias por la supuesta violación de las suposiciones de regresión al utilizar como variables independientes a los promedios por ambiente de la variable de respuesta.

Cruz (1989) indica que para la aplicación correcta del método de Eberhart y Russell, primero debe de efectuarse la prueba exacta de la interacción genotipo-ambiente. El mismo autor muestra que la significación de la interacción genotipo-ambiente depende del error ponderado del diseño de bloques al azar.

Otro método (aunque muy poco usado) para estudiar la interacción genotipo-ambiente es el de la ecovalencia propuesta por Wricke (1965).

En México se ha estudiado ]a interacción genotipoambiente en algodón (Palomo, 1992); maíz (Carballo, 1970); y girasol (Velázquez et al. (990), entre otros cultivos. Palomo (] 992) es quizá quién más ha estudiado el fenómeno en cuestión en algodonero.

En arroz, sólo se conocen tres estudios (Medina, 1991; Salcedo 1990 y Salcedo, 1992). El Primer autor estableció su trabajo en varias localidades arroceras del Sureste de la república Mexicana en condiciones de secano. En tanto Salcedo sus pruebas fueron conducidas en riego en el estado de Morelos, México.

De acuerdo con la literatura revisada (Alfonso $e t$ al. 1986; Alfonso et al. J 990; Fuchs y Pérez 1977; Deus et al. 1984; y Deus et al. 1990) en Cuba es donde se han lle- vado a cabo el mayor número de estudios sobre interacción genotipo-ambiente en el cultivo de arroz.

Conforme a lo anterior los objetivos del presente trabajo son:

Estudiar la estabilidad de las variables días a espigamiento (DE), altura de planta (AP) y rendimiento de grano $(R G)$ en líneas avanzadas y variedades comerciales de arroz.

Comparar los métodos de Eberhart y Russell vs Wricke en la estimación de la estabilidad de los genotipos para las variables probadas. Para esto sólo se considerará al Bi del primer método, ello con la finalidad de hacer más comparables dichos métodos.

Las hipótesis a confirmar son:

a.- Los genotipos ensayados son afectados en la misma proporción para cada variable por el ambiente de evaluación.

b.- No hay diferencia entre los métodos usados en el estudio de la interacción genotipo-ambiente.

\section{MATERIALES Y METODOS}

El estudio se efectuó durante Primavera-Verano de los años 1982 y 1983.

El material genético lo formaron cuatro líneas avanzadas, dos variedades de temporal y una variedad para condiciones de riego (Cuadro 1). El ensayo se estableció en cuatro localidades del estado de Campeche, México, éstas son: Nilchi, Edzná y Yohaltún (Cuadro 2), en esta última se repitió el experimento en Primavera-Verano 1983.

Las siembras de los experimentos se realizaron en el transcurso de la última semana del mes de Mayo, es decir antes de que se estableciera la temporada de lluvias.

El diseño experimental empleado en cada localidad fue el de bloques al azar con cuatro repeticiones. 
Cuadro 1. Descripción de los genotipos evaluados en el estudio de parámetros de estabilidad de arroz.

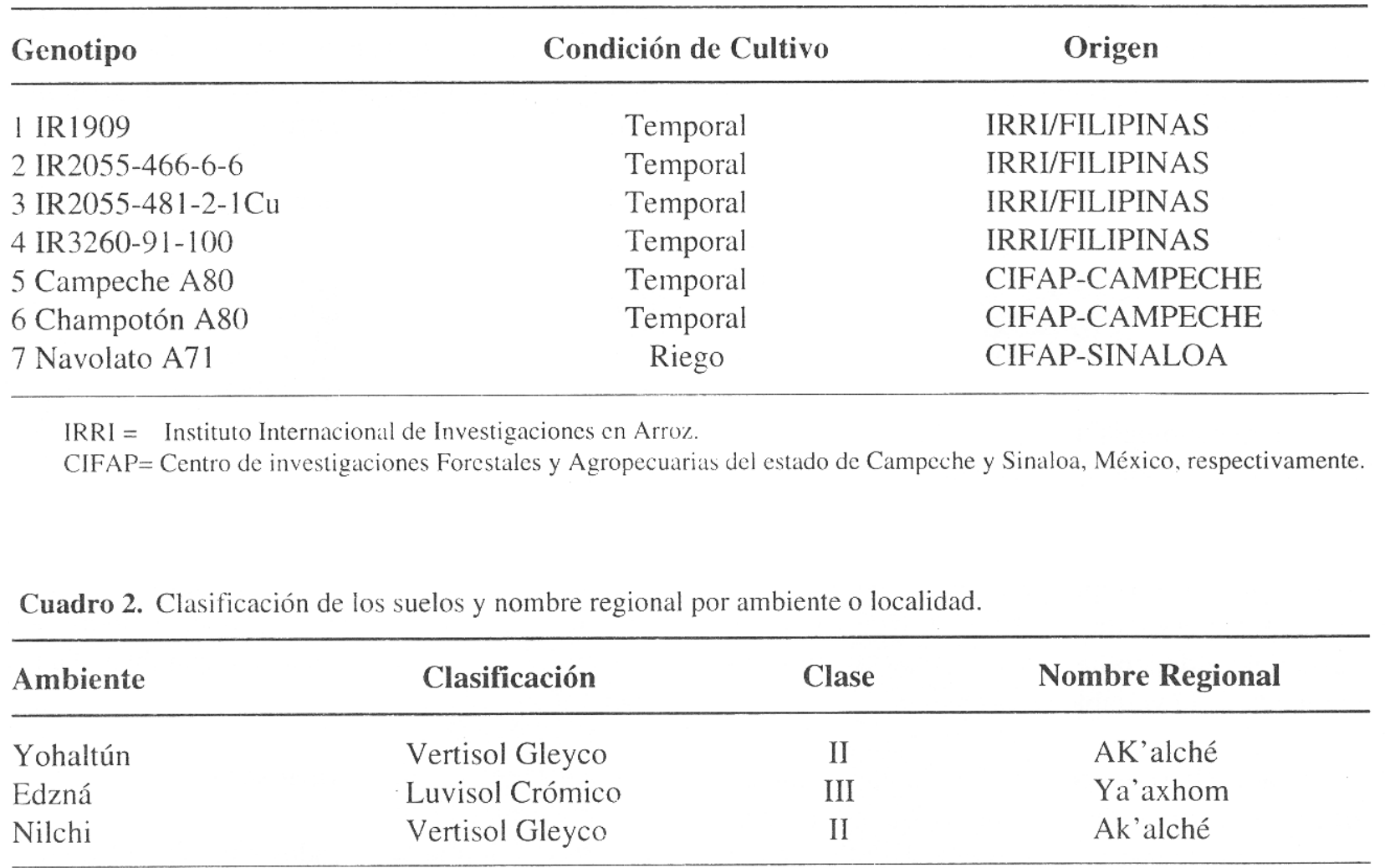

La siembra se realizó en hileras de 5,0 m de largo y $0,30 \mathrm{~m}$. entre cada una de ellas. El tamaño de la parcela experimental fue de $7,5 \mathrm{~m}^{2}$. La parcela útil fueron las tres hileras del centro de $4 \mathrm{~m}$ de longitud. Se usó una densidad de $120 \mathrm{~kg} / \mathrm{ha}$ de semilla de cada genotipo evaluado en cada localidad de prueba.

Las labores agrícolas, el control de maleza y plagas se llevaron a cabo como se indica en la guía para ]a producción de arroz públicada en el CIFAP-CAMPECHE.

Las variables que se midieron fueron:

a.- Días a Espigamiento (DE): Como el número de dias transcurridos desde la germinación hasta que el 50\% de las plantas en la parcela habían emitido la panícula.

b.- Altura de Planta (AP): Distancia en cm desde la base de la planta hasta la curvatura de la panícula. c.- Rendimiento de Grano (RG): Se cosecharon las tres hileras centrales, 40 días después de la emisión de la panícula y el rendimiento obtenido se expresó en $\mathrm{kg} / \mathrm{ha}$ ajustado al $14 \%$ de humedad.

Las tres variables medidas se estudiaron mediante análisis de estabilidad de Eberhart y Russell (1966) y el de ecovalencia de Wricke (1965).

El modelo estadístico para el análisis de variación combinado es el siguiente:

$$
Y i j k=M+A i+R / A i j+G k+G A i k+e i j k
$$

donde: $\mathrm{i}=1,2, \ldots . \mathrm{a} ; \quad \mathrm{j}=1,2, \ldots \ldots . . \mathrm{r} ; \quad \mathrm{k}=1,2, \ldots \ldots \ldots . \mathrm{g}$; $Y i j k=$ Observación del k-ésimo genotipo en el j-ésimo bloque del i-ésimo ambiente.

M: Media general.

A $i$ : Efecto del i-ésimo ambiente. 
$\mathrm{R} / \mathrm{A} i j=$ Efecto del j-ésimo bloque anidado en el i-ésimo ambiente.

$\mathrm{G} k=$ Efecto del k-ésimo genotipo.

GAij= Efecto de la interacción ambiente.

eijk= Error asociado a Yijk.

El modelo de Eberhart y Russell (ER) para estimar la estabilidad es:

$\mathrm{Y} i j k=\mathrm{M} i+\mathrm{B} i+\mathrm{I} j+\mathrm{S} i j(2)$

donde:

y $i j=$ Media de la i-ésima variedad en elj-ésimo ambiente.

$\mathrm{M} i=$ Comportamiento promedio de la i-ésima variedad en todos los ambientes.

$\mathrm{B} i=$ Coeficiente de regresión que mide la respuesta de la i-ésima variedad en el j-ésimo ambiente.

$\mathrm{I} j=$ Indice ambiental obtenido como la media de todas las variedades en el j-ésimo ambiente, menos la media general.

Sij= Desviación de regresión de la i-ésmia variedad en el j-ésimo ambiente.

El modelo de Wricke (Wi) para estudiar la estabilidad mediante ecovalencia se presenta como:

$\mathrm{W} i=\mathrm{S} i(\mathrm{Y} i j-\mathrm{Y} i .-\mathrm{Y} . j+\mathrm{Y} . .)^{2}$

donde:

W $i=$ Representa el valor de la ecovalencia del $i$-ésimo genotipo.

$\mathrm{S} i=$ Sumatoria de los i valores del $i$-ésimo genotipo.

Y $i j=$ Rendimiento promedio del $i$-ésimo genotipo en el $j$-ésimo ambiente.

Y $i=$ Desviaciones genotípicas.

$\mathrm{Y} j=$ Desviaciones ambientales.

Y..= Media general.

La significación de la ecovalencia de dos genotipos cualquiera, se hace mediante la prueba de F. El cociente que resulte de las dos ecovalencias confrontadas $(\mathrm{Fc}=(\mathrm{V} 2 / \mathrm{V} 1)$, se compararácon el valor de $\mathrm{Ft}$. El número de grados de libertad (gl) para esta prueba se calcula como sigue:

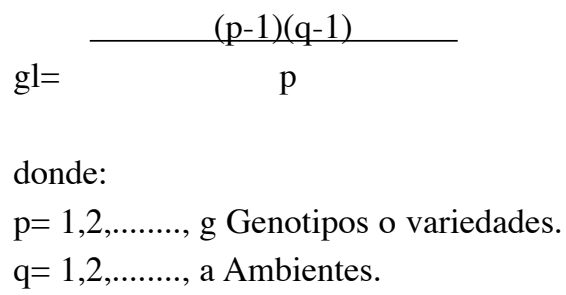

\section{RESULTADOS Y DISCUSION}

Al efectuar el análisis de variación combinado (modelo 1), se encontró significación en la interacción GA (Cuadro 3) en las tres variables estudiadas (DE, AP y RG). De acuerdo con estos resultados los genotipos evaluados se comportaron en forma diferente en los ambientes de evaluación, así como entre ellos mismos.

En base a ello se procedió a llevar a cabo el análisis de la regresión con el método de ER. Para ello se usó el paquete PARAM generado por Ortega y Magaña (1992). Los resultados de ese análisis se pueden observar en el Cuadro 4. En este caso, mirése que GA (lineal) sólo presentó significación para DE, no así para AP y RG. La no significación de GA (lineal) en AP y RG indica que los genotipos evaluados presentan comportamiento estadístico similar entre ellos en todos los ambientes de prueba.

Lo anterior es concomitante con lo que apunta Cruz (1989), es decir la significancia o no significación depende en mucho del error ponderado.

El Cuadro 5 muestra las medias para cada variable. De acuerdo con dicha información, para DE el genotipo Navolato A 71 fue el más precoz, en tanto que IR 1909 fue el más tardío. Ambos genotipos en base a su promedio son aceptables para condiciones de temporal, porque con una buena programación de siembra de estos, se puede escapar a los períodos de sequía, tan frecuentes en las localidades en donde se siembra arroz de temporal en el estado de Campeche. 
Cuadro 3. Cuadrados medios y significación de las variables estudiadas en líneas y variedades de arroz.

\begin{tabular}{lcccc}
\hline & \multicolumn{4}{c}{ CM } \\
\cline { 2 - 5 } FV & gl & DE & AP & RG \\
\hline A & 3 & $7425,70 * *$ & $1330,63 * *$ & $52991206,9 * *$ \\
R/A & 12 & 27,21 & 32,86 & 846664,9 \\
G & 6 & $215,46 * *$ & $191,36 * *$ & $3534703,9 * *$ \\
GA & 18 & $59,97 * *$ & $137,32 * *$ & $873614,2 * *$ \\
EE & 72 & 19,44 & 47,69 & 455809,2 \\
Total & 111 & & & \\
\hline
\end{tabular}

** = Significativa a $\mathrm{P}<0,01$

Cuadro 4. Análisis de variación de parámetros de estabilidad para DE, AP y RG en Arroz.

\begin{tabular}{lccc}
\hline & \multicolumn{3}{c}{ CM } \\
\cline { 2 - 4 } FV & DE & AP & RG \\
\hline G & $53,86^{*}$ & $47,85 \mathrm{NS}$ & $967230,69 *$ \\
A & 277,90 & 76,90 & 1991875,60 \\
A (lineal) & 5569,30 & 997,90 & 37561850,80 \\
GA (lineal) & $34,57 *$ & $43,39 \mathrm{NS}$ & $182174,16 \mathrm{NS}$ \\
Des. Pond. & 4,46 & 25,50 & 226746,92 \\
IR1909 & $0,24 \mathrm{NS}$ & $10,18 \mathrm{NS}$ & $592058,22 *$ \\
IR2055-466-6-6 & $0,37 \mathrm{NS}$ & $1,10 \mathrm{NS}$ & $18927,78 \mathrm{NS}$ \\
IR2055-481-2-1Cu & $3,61 \mathrm{NS}$ & $31,93 \mathrm{NS}$ & $43150,75 \mathrm{NS}$ \\
IR3260-91-100 & $3,12 \mathrm{NS}$ & $17,17 \mathrm{NS}$ & $94747,51 \mathrm{NS}$ \\
Campeche A80 & $5,25 \mathrm{NS}$ & $46,71 *$ & $466607,24 *$ \\
Champotón A80 & $12,65 \mathrm{NS}$ & $38,75 *$ & $141701,95 \mathrm{NS}$ \\
Navolato A71 & $6,00 \mathrm{NS}$ & $32,96 \mathrm{NS}$ & $330052,79 \mathrm{NS}$ \\
E. Pond. & 4,90 & 11,9 & 125706,10 \\
\hline
\end{tabular}

$*=$ Significativa a $\mathrm{P}<0,05$

NS $=$ No significativa

Des. Pond. $=$ Desviación Ponderada

E. Pond. $=$ Error Ponderado 
Cuadro 5. Medias de las variables evaluadas en los siete de Arroz.

\begin{tabular}{lccc}
\hline & \multicolumn{3}{c}{ VARIABLE } \\
\cline { 2 - 4 } Genotipo & $\mathbf{D E}$ & $\mathbf{A P}$ & $\mathbf{R G}$ \\
& $\mathbf{M}$ & $\mathbf{M}$ & $\mathbf{M}$ \\
\hline IR1909 & 114,9 & 75,6 & 3776,5 \\
IR2055-466-6-6 & 114,3 & 73,3 & 4566,8 \\
IR2055-481-2-1Cu & 113,9 & 75,0 & 4539,5 \\
IR3260-91-100 & 114,6 & 73,9 & 3820,8 \\
Campeche A80 & 113,8 & 75,7 & 4014,9 \\
Champoton A80 & 110,9 & 83,6 & 3181,4 \\
Navolato A71 & 104,7 & 74,9 & 3662,8 \\
\hline
\end{tabular}

$\mathrm{M}=$ media de las variables en cada genotipo

El resto de variables ya fueron definidas

Respecto a AP, dos fueron las líneas de menor altura (IR2055-466-e IR3260-91-100) y la más alta fue Champoton A80. Para esta variable, todos los genotipos evaluados muestran AP no muy propia para condiciones de secano. Sin embargo en esta variable (AP) la localidad de Ednzáfue la que tuvo el mayor efecto de reducción, ya que el tipo de suelo que es no hidromórfico, jugo un papel muy importante en la retención de humedad lo que afectó la altura de planta de todos los genotipos evaluados.

En cuanto a RG hubo mayor variabilidad entre los genotipos. El más alto rendimiento fue presentado por las líneas hermanas IR2055- 466-6-6 e IR2055-481-2-1 Cu. En tanto que los más bajos rendimientos fueron de Naval ato A 71 y Champotón A80. Esto era de esperarse ya que el primer genotipo se liberó para condiciones de riego y el segundo no obstante que es de temporal, presenta susceptibilidad a piricularia en el cuello de la panícula.

Los resultados obtenidos para los coeficientes de regresión (Bi) y ecovalencia (Wi), se consignan en el Cuadro 6. Obsérvese en el cuadro referido que para la variable $\mathrm{DE}$ sólo tres genotipos mostraron valores de $\mathrm{Bi}$ diferentes de 1,0. Mientras que para Wi el valor estimado con $9 \mathrm{gl}$ dió un valor de $\mathrm{F}$ en tablas de 9,3. Con ello se encontró que los genotipos 2, 3 y 4 son diferentes estadísticamente del resto en el valor de su ecovalencia. Al comparar ambos parámetros ( $\mathrm{Bi}$ vs $\mathrm{Wi}$ ) se puede ver que para el primero los valores de cada uno de los genotipos son muy cercanos a 1,0. En tanto que para Wi son (genotipos 2,3, y 4) los que presentan más fuerte ecovalencia es decir son los de valor más pequeño de Wi. Así mismo se puede apreciar que los rangos para cada genotipo en cada parámetro concuerdan en cierta medida en cuanto a su orden. Para AP sólo el genotipo IR2055-466-6-6 presentó un Bi mayor a 1.0. Para Wi no hubo significación para dicha variable entre genotipos.

Al comparar los rangos para ambos métodos, se encontró que la tendencia de la clasificación fue de que a menor valor de Bi el de Wi fue mayor o débil ecovalencia. Es decir, si el genotipo se comportó inestable de acuerdo a Bi, también lo fue conWi.

Lo descubierto para la variable RG fue que aquellos genotipos con valores de $\mathrm{Bi}$ alrededor de 1,0 tuvieron fuerte eco valencia o menores valores de Wi. No obstante al hacer la comparación en cuanto a rangos se notó que en la mayoría de los casos no coincidieron los de Bi con Wi, 
Cuadro 6. Coeficientes de regresión y ecovalencia de los genotipos evaluados de Arroz.

\begin{tabular}{|c|c|c|c|c|c|c|c|c|c|c|c|c|}
\hline \multirow[b]{3}{*}{ Genotipo } & \multicolumn{10}{|c|}{ VARIABLE } & \multirow[b]{3}{*}{ Wi } & \multirow[b]{3}{*}{$\mathbf{R}$} \\
\hline & \multicolumn{4}{|c|}{ DE } & \multicolumn{4}{|c|}{$\mathbf{A P}$} & \multicolumn{2}{|c|}{ RG } & & \\
\hline & $\mathrm{Bi}$ & $\mathbf{R}$ & Wi & $\mathbf{R}$ & $\mathbf{B i}$ & $\mathbf{R}$ & Wi & $\mathbf{R}$ & $\mathbf{B i}$ & $\mathbf{R}$ & & \\
\hline $\begin{array}{l}\text { IR1909 } \\
\text { IR2055-466 }\end{array}$ & $1,22 *$ & 7 & 37,4 & 5 & 1,31 & 6 & 33,8 & 1 & 0,98 & 4 & 1,31 & 6 \\
\hline $\begin{array}{l}-6-6 \\
\text { IR2055-481- }\end{array}$ & $1,11^{*}$ & 5 & 10,3 & 3 & $1,88^{*}$ & 7 & 113,3 & 5 & 0,99 & 5 & 0,09 & 1 \\
\hline $\begin{array}{l}2-1 \mathrm{Cu} \\
\text { IR3260-91- }\end{array}$ & 1,03 & 4 & 7,8 & 2 & 1,18 & 5 & 68,4 & 3 & 0,84 & 2 & 0,24 & 3 \\
\hline $\begin{array}{l}100 \\
\text { Campeche }\end{array}$ & 0,98 & 3 & 6,6 & 1 & 0,15 & 1 & 137,1 & 7 & 0,96 & 3 & 0,20 & 2 \\
\hline $\begin{array}{l}\text { A80 } \\
\text { Champotón }\end{array}$ & 1,18 & 6 & 36,8 & 4 & 0,56 & 2 & 120,1 & 6 & 1,36 & 7 & 1,62 & 7 \\
\hline $\begin{array}{l}\text { A80 } \\
\text { Navolato }\end{array}$ & 0,87 & 2 & 38,0 & 6 & 0,90 & 3 & 78,7 & 4 & 0,80 & 1 & 0,50 & 5 \\
\hline A71 & $0,61 *$ & 1 & 132,6 & 7 & 1,01 & 4 & 66,0 & 2 & 1,08 & 6 & 0,49 & 4 \\
\hline
\end{tabular}

$\mathrm{R}=$ Rango o posición de acuerdo a su valor

excepto para el cultivar Campeche A80 que fue el que presentó el más alto valor de Bi y la más débil ecovalencia Wi.

De acuerdo con esto, se puede establecer que a medida que el ambiente es mejor, el citado cultivar incrementa su rendimiento. Sin embargo estos resultados se contraponen con lo encontrado por Salcedo (1990), el observó en su estudio que en condiciones de riego el cultivar Campeche A80 responde mejor en ambientes pobres, esto hace suponer que quizá para temporal serían buenas condiciones. Otra posible explicación es que Campeche A80 se liberó para condiciones de secano.

Para tener una mejor comparación de ambos métodos sería conveniente que los coeficientes Bi y Wi, así como los $\mathrm{Si}$ (desviación) se correlacionarano Con este análisis puede indicarse con más precisión el tipo de asociación que existe entre ambas metodologías (Deus et al. 1986).

De acuerdo con los resultados encontrados en este estudio, las conclusiones que se pueden establecer son:
En forma general los genotipos se comportaron estables en los diferentes ambientes de evaluación para las tres variables estudiadas (DE, AP y RG). Encontrándose que para DE los genotipos IR 1909 e IR2055-466-6-6, respondieron en forma positiva a medida que el ambiente de evaluación fue mejor. El segundo genotipo también mostró esta respuesta para la variable AP.

Los métodos de ER y Wi son efectivos para el estudio de la estabilidad de genotipos de arroz. Sin embargo es necesario hacer correlación entre los coeficientes de uno y otro método para determinar si existe o no asociación entre ellos. De resultar positiva y significativa la asociación de $\mathrm{Bi}$, Si y Wi, es indistino usar cualquier metodología puesto que es de esperarse obtener los mismos resultados. Las líneas IR2055-466-6-6 e IR2055-481-2-1 $\mathrm{Cu}$ pueden ser liberadas como nuevas variedades mejoradas con muchas posibilidades de éxito en siembras de secano.

De acuerdo con lo anterior las hipótesis planteadas en la presente prueba se aceptan. 


\section{BIBLIOGRAFIA}

ALFONSO,R.; DEUS, J. E.; MARTI, F. 1986. Estabilidad en el rendimiento y el ciclo de cinco cultivares de arroz (Oryza sativa L.). Agrotecnia de Cuba (Cuba) 18(1):7-11.

ALFONSO, R.; DEUS, J. E. SUÁREZ, E. 1988. Estimación de la interacción genotipo-ambiente y heredabilidad de algunos caracteres agronómicos en arroz (Oryza sativa L.). Agrotecnia de Cuba (Cuba) 20(1):7-11.

ALFONSO, R.; DEUS, 1. E.; SUÁREZ, E.; DUARDO, T. 1990. Estabilidad para el rendimiento y el ciclo en 11 cultivares de arroz (Oryza sativa L.) y repetibilidad de estos caracteres. Agrotecnia de Cuba (Cuba) 22(1):7-11.

CARBALLO, C. A. 1970. Comparación de variedades de maíz del bajío y de la Mesa Central por su rendimiento y estabilidad. Tesis de Maestría. Colegio de Postgraduados. ENA. Chapingo, México. 88p.

CRUZ, M, R. 1989. Un ejemplo de la prueba exacta de los parámetros de estabilidad de Eberhart y Russell. Fitotecnia (Méx.). 12(2):147-155.

CRUZ, M. R.; SALAZAR,G. M. 1992. Métodos alternativos en el análisis de la interacción genotipo-ambiente. Memorias Simposio Interacción Genotipo-Ambiente en genotecnia vegetal. Publicado por SOMEFI, Guadalajara, Méx. pp. 127-148.

DEUS, J. E.; AVILA, J.; PÉREZ, R,; LAZO, E.; RODRÍGUEZ, S.; AMADOR, M. 1984. Interacción genotipo-ambiente y heredabilidad en algunos caracteres de importancia agronómica en arroz (Oryza sativa L.). Agrotecnia de Cuba (Cuba)16(2):77-87.

DEUS, J. E.; PÉREZ, R.; AVILA, J.; RODRÍGUEZ, S. 1990. Análisis de correlaciones genotípicas, fenotípicas y ambientales entre el rendimiento y caracteres de importancia agronómica en arroz (Oryza sativa L.). Agrotecnia de $\mathrm{Cu}$ ba (Cuba) 22(1):51-56.

EBERHART, S. A.; RUSSELL, W. A. 1966. Stability parameters forcomparing varieties. Crop Sci. (USA) 6:36-40.
FINLAY, K. W.; WILKINSON, G. N. 1963. The analysis of adaptation in a plant-breeding programme. Aust. J. Agric. Res. (Aust.). 14(6):742-754.

FUCHS, A.; PÉREZ, P. J. 1977. Determinación del rendimiento en experimentos con variedades y sus descripciones en un ejemplo con arroz. Centro Agrícola (Cuba) 93-107. septiembre-diciembre.

MEDINA, M. J. 1991. Determinación de parámetros de estabilidad en 25 genotipos de arroz (Oryza sativa L.) de temporal en el Sureste de México. Tesis de Lic. Chiná, Campeche, (Méx.). 59p. ITA 5.

ORTEGA, A. J.; MAGAÑA, T. O. S. 1992. Análisis, Diseño y Programación. Parámetros de estabilidad en base al modelo propuesto por Eberhart y Russell. Texcoco (Méx.).

PALOMO, G. A. 1992. Estudios de interacción genético ambiental en el cultivo de algodonero. Memorias Simposio interacción Genotipo-Ambiente en genotecnia vegetal. Publicado por SOMEFI, Guadalajara, Jal (Méx.). pp 326350.

PÉREZ, P. J.; ORELLANA, P.; MARTÍNEZ, J.; PÉREZ, P. R.; AVILA, J.; MURGA, E.; RÍG RODRÍGUEZ, S. 1977. Influencia de las épocas de siembra y las regiones sobre el ciclo y rendimiento en arroz (Oryza sativa L.). Agrotecnia de Cuba (Cuba) 9(2):37-47.

SALCEDO, A. J. 1992. Comparación de líneas y variedades de arroz (Oryza sativa L.) por rendimiento y estabilidad. XIV. Congreso Nacional de Fitogenética. Publicado por SOMEFI, Tuxtla Gutierrez, Chis, (Méx.). p244.

SALCEDO, A. J. 1990. Estabilidad del rendimiento en arroz (Oryza sativa L.) en Morelos. XIII. Congreso Nacional de Fitogenética. Publicado por SOMEFI, Cd. Juárez, Chih, (Méx.). p257.

VELAZQUEZ, C. M.; CARBALLO, C. A.; RINCÓN, C. J. J.; 1992. Estabilidad de genotipos de girasol (Helianthus annus L.) en el Valle de México. Chapingo (Méx.). Año XV (71-72):79-83.

YATES, F.; COCHRAN, G. A. 1938. The analysis of group of experiments. J. Agric. Sci. (Ing.) 23:556-580. 